\title{
Comparative Analysis of Different Imputation Methods to Treat Missing Values in Data Mining Environment
}

\author{
Rahul Singhai \\ IIPS, Devi Ahilya University \\ Indore, India
}

\begin{abstract}
Data cleaning is one of the important step of KDD (Knowledge discovery in database) process. One critical problem in data cleaning is the presence of missing values. Various approaches have proposed to find \& replace such missing data including use of mean value, use of global constant, replace by more probable value etc. Imputation is one of the important procedures in statistics that is used to replace the missing values in a data set. One advantage of this approach is that the missing data treatment is independent of the learning algorithms that are used. This allows the user to select the most suitable and appropriate imputation method for each situation. This paper analyze the six different imputation methods proposed in the field of statistics and implement them in Data mining environment. An artificial data set of 1000 records is used to analyze the performance of these methods. For testing the significance of these methods Z-test approach were used. Exhaustive experiments show the effectiveness of the proposed methods. It is assumed that all the attributes of input data are of numeric data type.
\end{abstract}

\section{Keywords}

KDD, Data mining, Imputation methods, Data pre-processing, sampling, attribute missing values.

\section{INTRODUCTION}

Missing value treatment is another critical issue in data mining. If the information repository on which data mining methods are applied to extract patterns, contains some missing values, then obviously the quality of the pattern extracted may be degraded or poor. Imputation is a promising method used to find \& replace the missing values where data set attributes are highly associated to each other. Thus, through the identification of dependency among attributes, missing values can be determined. The objective of this paper is to propose the different imputation methods to improve the quality of KDD process and to compare \& analyze the performance of these methods using Z-test in a large database, so that the best possible methods could be proposed in data mining.

\section{IMPUTATION METHODS FOR MISSING DATA TREATMENT USING AUXILIARY INFORMATION}

Several imputation techniques are described by different researchers, some of them are better over others. Rubin (1976) addressed three concepts: MAR (missing at random), OAR (observed at random) and PD (parametric distribution). In what follows MCAR (missing completely at random) is used. Let $\bar{Y}=N^{-1} \sum_{i=1}^{N} Y_{i}$ be the mean of a finite data set under consideration for estimation. A simple random sample $\mathrm{S}$ without replacement (SRSWOR), of size $\mathrm{n}$ is drawn from data set $\Omega=\{1,2, \ldots, N\}$ to estimate $\bar{Y}$. The sample $\mathrm{S}$ of $\mathrm{n}$ units contains $\mathrm{r}$ responding units $(r<n)$ forming a set $\mathrm{R}$ and $(\mathrm{n}-$ r) non-responding with the sub-space $(\mathrm{n}-\mathrm{r})$ having symbol $R^{C}$ in the space. The attribute $\mathrm{Y}$ is of main interest and $\mathrm{X}$ an auxiliary attribute correlated with $\mathrm{Y}$. For every unit $i \in R$, the value $y_{i}$ is observed available. However, for the units $i \in R^{C}$, the $y_{i}$ values are missing and imputed values are to be derived. The ith value $x_{i}$ of auxiliary e is used as a source of imputation for missing data when $i \in R^{C}$. This is to assume that for sample $\mathrm{S}$, the data $x_{s}=\left\{x_{i}: i \in S\right\}$ are known and $S=R \cup R^{C}$. The following figure shows the diagrammatic representation of this sampling procedure.

\section{Data warehouse}

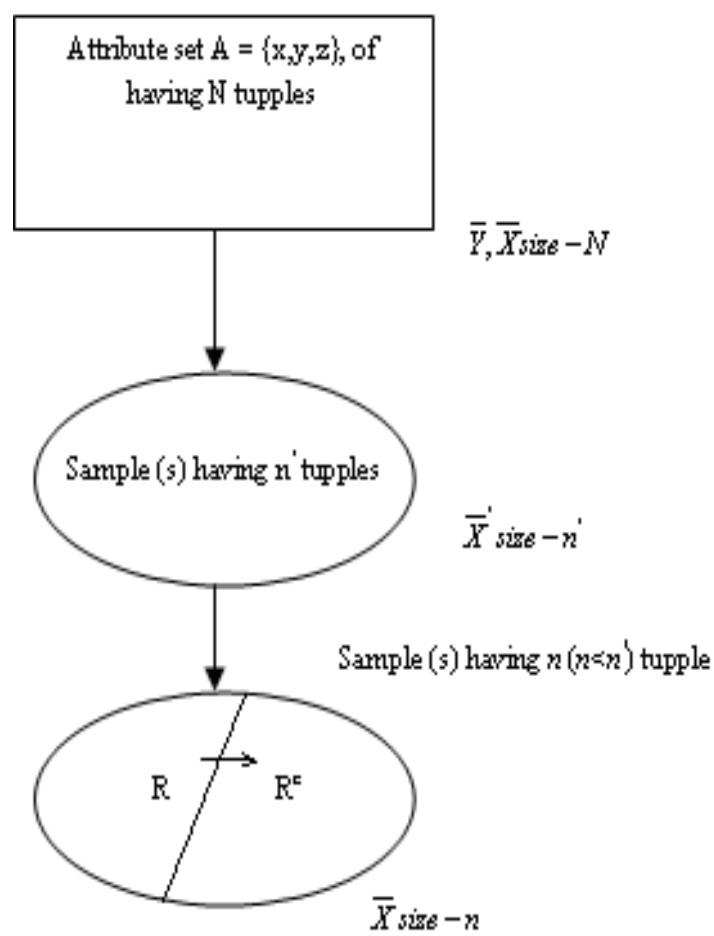

Under this mentioned setup, some of the well known imputation methods, that can be used in data mining are given below :

\subsection{Mean Method of Imputation}

For $y_{i}$ define $y_{\bullet i}$ as 


$$
y_{\bullet i}=\left\{\begin{array}{llc}
y_{i} & \text { if } & i \in R \\
y_{r} & \text { if } & i \in R^{C}
\end{array}\right.
$$

(ii) : $\quad M\left(\bar{y}_{\text {СОMP }}\right)=\left(\frac{1}{r}-\frac{1}{N}\right) \bar{Y}^{2} C_{Y}^{2}$

$$
+\left(\frac{1}{r}-\frac{1}{n}\right) \bar{Y}^{2}\left[(1-\alpha)^{2} C_{X}^{2}-2(1-\alpha) \rho C_{Y} C_{X}\right]
$$

$\bar{Y}$ is :

$$
\bar{y}_{m}=\frac{1}{r} \sum_{i \in R} \bar{y}_{i}=\bar{y}_{r}
$$

\subsection{Ratio Method of Imputation}

For sampled values $y_{i}$ and $x_{i}$ define $y_{\bullet i}$ as

$$
\begin{gathered}
y_{\bullet}=\left\{\begin{array}{lll}
y_{i} & \text { if } & i \in R \\
\hat{b} x_{i} & \text { if } & i \in R^{C}
\end{array}\right. \\
\text { where } \hat{b}=\sum_{i \in R} y_{i} / \sum_{i \in R} x_{i}
\end{gathered}
$$

Using above, the imputation-based estimator of data set mean $\bar{Y}$ is:

$$
\begin{gathered}
\bar{y}_{S}=\frac{1}{n} \sum_{i \in S} \bar{y}_{\bullet i}=\bar{y}_{r}\left(\frac{\bar{x}_{n}}{\bar{x}_{r}}\right)=\bar{y}_{R A T} \\
\text { where } \bar{y}_{r}=\frac{1}{r} \sum_{i \in R} y_{i}, \quad \bar{x}_{r}=\frac{1}{r} \sum_{i \in R} x_{i} \text { and } \\
\bar{x}_{n}=\frac{1}{n} \sum_{i \in S} x_{i}
\end{gathered}
$$

\subsection{Compromised Method of Imputation}

Singh and Horn (2000) proposed compromised imputation procedure

$$
y_{\bullet i}= \begin{cases}(\alpha n / r) y_{i}+(1-\alpha) \hat{b} x_{i} & \text { if } \quad i \in R \\ (1-\alpha) \hat{b} x_{i} & \text { if } \quad i \in R^{C}\end{cases}
$$

where $\alpha$ is a suitably chosen constant, such that the resultant variance of the estimator is minimum. The imputation-based estimator, for this case, is

$$
\bar{y}_{\text {COMP }}=\left[\alpha \bar{y}_{r}+(1-\alpha) \bar{y}_{r} \frac{\bar{x}_{n}}{\bar{x}_{r}}\right]
$$

Lemma : The bias, m.s.e. and minimum m.s.e. of $\bar{y}_{\text {COMP }}$ is [As per Singh and Horn (2000)]:

(i) $B\left(\bar{y}_{\text {СOMP }}\right)=(1-\alpha)\left(\frac{1}{r}-\frac{1}{n}\right) \bar{Y}\left(C_{X}^{2}+\rho C_{Y} C_{X}\right)$ (iii) : $\quad$ For optimum $\alpha=\left(1-\rho \frac{C_{Y}}{C_{X}}\right)$, the minimum m.s. e. of $y_{\text {COMP }}$ is given by the expression

$$
M\left(\bar{y}_{C O M P}\right)_{\min }=\left[\left(\frac{1}{r}-\frac{1}{N}\right)-\left(\frac{1}{r}-\frac{1}{n}\right) \rho^{2}\right] S_{Y}^{2}
$$

\subsection{Ahmed Methods of Imputation}

For the case where ${ }^{y_{j i}}$ denotes the ith available observation for the jth imputation method. Ahmed et al. (2006) suggested the following:

(A):

$$
y_{1 i}=\left\{\begin{array}{l}
y_{i} \quad \text { if } \quad i \in R \\
\frac{1}{(n-r)}\left[n \bar{y}_{r}\left(\frac{\bar{X}}{\bar{x}_{n}}\right)^{\beta_{1}}-r \bar{y}_{r}\right] \quad \text { if } \quad i \in R^{C}
\end{array}\right.
$$

Under this, the point estimator of $\bar{Y}$ is

$$
t_{1}=\bar{y}_{r}\left(\frac{\bar{X}}{\overline{x_{n}}}\right)^{\beta_{1}}
$$

\section{Lemma :}

(i) The bias of $t_{1}$ is :

$$
B\left(t_{1}\right)=\left(\frac{1}{n}-\frac{1}{N}\right) \bar{Y}\left[\frac{\beta_{1}\left(\beta_{1}+1\right)}{2} C_{x}^{2}-\beta_{1} \rho C_{Y} C_{X}\right]
$$

(ii) The m.s.e. of $t_{1}$ is :

$$
M\left(t_{1}\right)=\bar{Y}^{2}\left[\left(\frac{1}{r}-\frac{1}{N}\right) C_{Y}^{2}+\left(\frac{1}{n}-\frac{1}{N}\right) \beta_{1}^{2} C_{x}^{2}-\left(\frac{1}{n}-\frac{1}{N}\right) 2 \beta_{1} \rho C_{Y} C_{X}\right]
$$

(iii) The minimum m.s.e. of $t_{1}$ is :

$$
M\left(t_{1}\right)_{\min }=\left(\frac{1}{r}-\frac{1}{N}\right) S_{Y}^{2}-\left(\frac{1}{n}-\frac{1}{N}\right) \frac{S_{X Y}^{2}}{S_{X}^{2}}
$$


for the optimum value of $\beta_{1}$ which is given by $\beta_{1}=\rho \frac{C_{Y}}{C_{X}}$.

(B) $y_{2 i}=\left\{\begin{array}{lll}y_{i} & \text { if } & i \in R \\ \frac{1}{(n-r)}\left[n \bar{y}_{r}\left(\frac{\bar{x}_{n}}{\bar{x}_{r}}\right)^{\beta_{2}}-r \bar{y}_{r}\right] & \text { if } & i \in R^{C}\end{array}\right.$

Under this, the point estimator of $\bar{Y}$ is

$$
t_{2}=\bar{y}_{r}\left(\frac{\bar{x}_{n}}{\bar{x}_{r}}\right)^{\beta_{2}}
$$

\section{Lemma}

(iv) The bias of $t_{2}$ is :

$$
B\left(t_{2}\right)=\left(\frac{1}{r}-\frac{1}{n}\right) \bar{Y}\left[\frac{\beta_{2}\left(\beta_{2}+1\right)}{2} C_{x}^{2}-\beta_{2} \rho C_{Y} C_{X}\right]
$$

(v) The M.S.E. of $t_{2}$ is :

$M\left(t_{2}\right)=\bar{Y}^{2}\left[\left(\frac{1}{r}-\frac{1}{N}\right) C_{Y}^{2}+\left(\frac{1}{r}-\frac{1}{n}\right) \beta_{2}^{2} C_{x}^{2}-\left(\frac{1}{r}-\frac{1}{n}\right) 2 \beta_{2} \rho C_{Y} C_{X}\right]$

(vi) The minimum m.s.e. of $t_{2}$ is:

$$
M\left(t_{2}\right)_{\min }=\left(\frac{1}{r}-\frac{1}{N}\right) S_{Y}^{2}-\left(\frac{1}{r}-\frac{1}{n}\right) \frac{S_{X Y}^{2}}{S_{X}^{2}}
$$

for the optimum value of $\beta_{2}$ which is given by $\beta_{2}=\rho \frac{C_{Y}}{C_{X}}$

(C)

$$
y_{3 i}=\left\{\begin{array}{lll}
y_{i} & \text { if } \quad & i \in R \\
\frac{1}{(n-r)}\left[n \bar{y}_{r}\left(\frac{\bar{X}}{\bar{x}_{r}}\right)^{\beta_{3}}-r \bar{y}_{r}\right] & \text { if } \quad & i \in R^{C}
\end{array}\right.
$$

Under this, the point estimator of $\bar{Y}$ is

$$
t_{3}=\bar{y}_{r}\left(\frac{\bar{X}}{\bar{x}_{r}}\right)^{\beta_{3}}
$$

The $\beta_{1}, \beta_{2}$ and $\beta_{3}$ are suitably chosen constants, so as to keep the variance of the resultant estimator minimum.
As special cases :

when $\beta_{3}=1$, then $t_{\text {Ratio }}=\bar{y}_{r}\left(\frac{\bar{X}}{\bar{x}_{r}}\right)$

and when $\beta_{3}=-1$, then $t_{\operatorname{Pr} \text { oduct }}=\bar{y}_{r}\left(\frac{\bar{x}_{r}}{\bar{X}}\right)$

This is natural analogue of the ratio estimator called the product estimator used when an auxiliary variate $x$ has negative correlation with $y$.

\section{Lemma}

(vii) The bias of $t_{3}$ is :

$B\left(t_{3}\right)=\left(\frac{1}{r}-\frac{1}{N}\right) \bar{Y}\left[\frac{\beta_{3}\left(\beta_{3}+1\right)}{2} C_{x}^{2}-\beta_{3} \rho C_{Y} C_{X}\right]$

(viii) The m.s.e. of $t_{3}$ is :

$M\left(t_{3}\right)=\bar{Y}^{2}\left(\frac{1}{r}-\frac{1}{N}\right)\left[C_{Y}^{2}+\beta_{3}^{2} C_{x}^{2}-2 \beta_{3} \rho C_{Y} C_{X}\right]$

(ix) The minimum m.s.e. of $t_{3}$ is :

$M\left(t_{3}\right)_{\min }=\left(\frac{1}{r}-\frac{1}{N}\right) S_{Y}^{2}\left(1-\rho^{2}\right)$

for the optimum value of $\beta_{3}$ which is given by $\beta_{3}=\rho \frac{C_{Y}}{C_{X}}$

\subsection{Factor-Type Methods of Imputation :}

For the case where ${ }^{y_{j i}}$ denotes the ith available observation for the jth imputation method. Shukla and Thakur (2008) suggested the following :

(D)

$$
\left(y_{F T 1}\right)_{i}= \begin{cases}y_{i} & \text { if } \quad i \in R \\ \frac{\bar{y}_{r}}{(n-r)}\left[n \phi_{1}(k)-r\right] \quad \text { if } \quad & i \in R^{C}\end{cases}
$$

(E) $\quad\left(y_{F T 2}\right)_{i}=\left\{\begin{array}{lll}y_{i} & \text { if } & i \in R \\ \frac{\bar{y}_{r}}{(n-r)}\left[n \phi_{2}(k)-r\right] & \text { if } & i \in R^{C}\end{array}\right.$ 
(F)

$$
\left(y_{F T 3}\right)_{i}=\left\{\begin{array}{lll}
y_{i} & \text { if } & i \in R \\
\frac{\bar{y}_{r}}{(n-r)}\left[n \phi_{3}(k)-r\right] & \text { if } & i \in R^{C}
\end{array}\right.
$$

where

$$
\begin{aligned}
& \phi_{1}(k)=\left[\frac{(A+C) \bar{X}+f B \bar{x}_{n}}{(A+f B) \bar{X}+C \bar{x}_{n}}\right] ; \\
& \phi_{2}(k)=\left[\frac{(A+C) \bar{x}_{n}+f B \bar{x}_{r}}{(A+f B) \bar{x}_{n}+C \bar{x}_{r}}\right] ; \\
& \phi_{3}(k)=\left[\frac{(A+C) \bar{X}+f B \bar{x}_{r}}{(A+f B) \bar{X}+C \bar{x}_{r}}\right] ;
\end{aligned}
$$

$A=(k-1)(k-2) ; B=(k-1)(k-4) ;$

$C=(k-2)(k-3)(k-4) ; f=\frac{n}{N}$ and $0<k<\infty$ is a

constant. Under (15), (16) and (17) the point estimators of $\bar{Y}$ are:

$$
\left.\begin{array}{l}
T_{F T 1}=\bar{y}_{r} \phi_{1}(k) \\
T_{F T 2}=\bar{y}_{r} \phi_{2}(k) \\
T_{F T 3}=\bar{y}_{r} \phi_{3}(k)
\end{array}\right\}
$$

The term $k(0<k<\infty)$ is a suitably chosen constant, such that the mean squared error of the resultant estimator is minimum.

(10) : Bias of $T_{F T 1}$ up to first order of approximation is :

$$
B\left(T_{F T 1}\right)=-P M_{2} \bar{Y}\left(\theta_{2} C_{X}^{2}-\rho C_{Y} C_{X}\right)
$$

(11) : Mean squared error of $T_{F T 1}$ up to first order is :

$$
M\left(T_{F T 1}\right)=\bar{Y}^{2}\left[M_{1} C_{Y}^{2}+P M_{2}\left(P C_{X}^{2}+2 \rho C_{Y} C_{X}\right)\right]
$$

(12) : The minimum m.s.e. of $T_{F T 1}$ occurs when $P=-V$ and expression is:

$$
M\left(T_{F T 1}\right)_{\min }=\left(M_{1}-M_{2} \rho^{2}\right) S_{Y}^{2}
$$

(13) : Bias of the estimator $T_{F T 2}$ is :

$$
B\left(T_{F T 2}\right)=-P M_{3} \bar{Y}\left(\theta_{2} C_{X}^{2}-\rho C_{Y} C_{X}\right)
$$

(14) : Mean squared error of $T_{F T 2}$ is :

$$
M\left(T_{F T 2}\right)=\bar{Y}^{2}\left[M_{1} C_{Y}^{2}+P M_{3}\left(P C_{X}^{2}+2 \rho C_{Y} C_{X}\right)\right]
$$

(15) : The minimum m.s.e. of $T_{F T 2}$ is at $P=-V$

$M\left(T_{F T 2}\right)_{\min }=\left(M_{1}-M_{3} \rho^{2}\right) S_{Y}^{2}$

(16) : Estimator $T_{F T 3}$ in terms of $\varepsilon, \delta$ and $\eta$ up to first order of approximation is :

$T_{F T 3}=\bar{Y}\left[1+\varepsilon+P\left(\delta+\varepsilon \delta-\theta_{2} \delta^{2}\right)\right]$

(17) : Bias expression is :

$B\left(T_{F T 3}\right)=-P M_{1} \bar{Y}\left(\theta_{2} C_{X}^{2}-\rho C_{Y} C_{X}\right)$

(18) : The mean squared error of $T_{F T 3}$ is :

$M\left(T_{F T 3}\right)=M_{1} \bar{Y}^{2}\left[C_{Y}^{2}+P^{2} C_{X}^{2}+2 P \rho C_{Y} C_{X}\right]$

(19) : Minimum mean squared error is:

$$
M\left(T_{F T 3}\right)_{\min }=\left(1-\rho^{2}\right) M_{1} S_{Y}^{2} \quad \text { at } P=-V
$$

\section{TESTING THE SIGNIFICANCE OF THE DIFFERENCE BETWEEN THE MEANS OF TWO LARGE SAMPLES:}

Suppose two random samples of $\mathrm{n} 1$ and $\mathrm{n} 2$ members respectively have been drawn from the same data set of standard deviation $\sigma$. since the difference of their means ( $\left.\bar{x}_{1} \sim \bar{x}_{2}\right)$ is due to fluctuations of sampling due to the assumption that the samples are independent and drawn from the same data set. The standard error e of the difference of their means is given by $e^{2}=e_{1}^{2}+e_{2}^{2}$, where e1 and e2 are the standard error of the means of the two samples and are

$$
\frac{\sigma}{\sqrt{n_{1}}} \text { and } \frac{\sigma}{\sqrt{n_{2}}} \text { respectively, so that } e=\sigma\left(\frac{1}{n_{1}}+\frac{1}{n_{2}}\right)^{1 / 2}
$$

If $\mathrm{n} 1$ and $\mathrm{n} 2$ be sufficiently large than $\bar{x}_{1} \sim \bar{x}_{2}$ is asymptotically normally distributed with mean zero and standard deviation e. Consequently, if the difference $\bar{x}_{1} \sim \bar{x}_{2}$ exceeds $3 e$ the difference can hardly be accounted for by the fluctuations of sampling and our assumption unlikely to be correct while if difference exceeds $2 \mathrm{e}$, it is regarded as significant at the $5 \%$ level of probability.

If two independent samples of $\mathrm{n} 1$ and $\mathrm{n} 2$ members respectively be drawn from different data sets with variances $\sigma_{1}^{2}$ and $\sigma_{2}^{2}$ respectively we can examine whether the two data sets from which samples have been drawn differ in mean apart from the difference in dispersion. Since the samples are 
independent the s.e. e of the difference of their means is given by $e^{2}=\left(\frac{\sigma_{1}^{2}}{n_{1}}+\frac{\sigma_{2}^{2}}{n_{2}}\right)$

Assuming that $\mathrm{n} 1$ and $\mathrm{n} 2$ are large and the two data sets have the same means, the difference of the means of the samples will be normally distributed with mean zero and s.d. e given by

If the difference of the means of the samples exceeds $3 \mathrm{e}$, it can hardly be accounted for on the basis of fluctuations of sampling and our assumption that the two data sets have the same mean is almost certainly wrong.

In the above discussion the following assumption have been considered:

1. It is assumed that the data set variance $\sigma_{1}^{2}, \sigma_{2}^{2}$ are known. In practice this is hardly the case and accordingly in the expressions for e, these have to be replaced by their estimated obtained for the samples, viz., by the sample variances $s_{1}^{2}$ and $s_{2}^{2}$ respectively,

where $s_{j}^{2}=\frac{1}{n_{j}} \sum_{i=1}^{n_{j}}\left(x_{i}-\bar{x}_{j}\right)^{2} j=1,2$.

2. The above tests are valid for only large samples for two reasons: (i) The parent data sets may not be normal, though we are assuming that they do not depart strikingly from it. In particular, we assume that the data sets of finite variances. For data sets like Cauchy's where the variance is not finite, the tests would break down completely even for infinitely large samples.

(ii) The data set variances are not known and have to be replaced by their estimates.

3. For normal data sets with known variances, the above tests are valid for all sample sizes.

4. If the hypothesis to be tested is that the data set means are $\mu$ and $\mu^{\prime}$, we can carry out the test of significance as above, but in this case

$$
z=\frac{\left(\bar{x}_{1}-\bar{x}_{2}\right) \sim\left(\mu-\mu^{\prime}\right)}{\sqrt{\frac{s_{1}^{2}}{n_{1}}+\frac{s_{2}^{2}}{n_{2}}}}
$$

will be asymptotically a standard normal variant for large $n_{1}, n_{2}$.

\section{EXPERIMENTAL ANALYSIS}

Table 1: Test of significance between $\bar{Y}$ (without missing) and $\bar{Y}$ (after imputation by mean method)

\begin{tabular}{|c|c|c|c|c|c|c|c|}
\hline S.No. & missing \% & $\bar{Y}$ & $\begin{array}{c}\text { S.D. } \\
\text { (without) }\end{array}$ & $\begin{array}{c}\bar{Y} \\
\text { (mean) }\end{array}$ & $\begin{array}{c}\text { S.D. } \\
\text { (mean) }\end{array}$ & S.E. & Z-test \\
\hline 1 & 1.00 & 42.4850 & 13.9677 & 42.5152 & 13.9622 & 0.3737 & -0.0807 \\
\hline 2 & 2.00 & 42.4850 & 13.9677 & 42.3061 & 13.7705 & 0.3724 & 0.4803 \\
\hline 3 & 4.00 & 42.4850 & 13.9677 & 42.5781 & 13.8953 & 0.3732 & -0.2495 \\
\hline 4 & 6.00 & 42.4850 & 13.9677 & 42.5638 & 13.3364 & 0.3695 & -0.2133 \\
\hline 5 & 8.00 & 42.4850 & 13.9677 & 42.5978 & 13.5484 & 0.3709 & -0.3042 \\
\hline 6 & 10.00 & 42.4850 & 13.9677 & 41.9667 & 13.2129 & 0.3687 & 1.4060 \\
\hline 7 & 12.00 & 42.4850 & 13.9677 & 42.8239 & 13.0783 & 0.3677 & -0.9215 \\
\hline 8 & 14.00 & 42.4850 & 13.9677 & 42.5523 & 13.0947 & 0.3678 & -0.1830 \\
\hline 9 & 16.00 & 42.4850 & 13.9677 & 42.6429 & 12.9134 & 0.3666 & -0.4306 \\
\hline 10 & 18.00 & 42.4850 & 13.9677 & 42.3537 & 12.7720 & 0.3656 & 0.3592 \\
\hline 11 & 20.00 & 42.4850 & 13.9677 & 41.7125 & 12.2484 & 0.3621 & 2.1337 \\
\hline 12 & 22.00 & 42.4850 & 13.9677 & 42.2115 & 12.2072 & 0.3618 & 0.7559 \\
\hline 13 & 24.00 & 42.4850 & 13.9677 & 42.4868 & 12.1750 & 0.3615 & $-\mathbf{0 . 0 0 5 1}$ \\
\hline 14 & 26.00 & 42.4850 & 13.9677 & 42.6284 & 11.9687 & 0.3601 & -0.3981 \\
\hline 15 & 28.00 & 42.4850 & 13.9677 & 42.6111 & 11.6830 & 0.3581 & -0.3521 \\
\hline 16 & 30.00 & 42.4850 & 13.9677 & 42.1643 & 11.1876 & 0.3546 & 0.9043 \\
\hline 17 & 32.00 & 42.4850 & 13.9677 & 42.5646 & 12.0429 & 0.3606 & -0.2208 \\
\hline 18 & 34.00 & 42.4850 & 13.9677 & 42.4091 & 10.4010 & 0.3491 & 0.2175 \\
\hline 19 & 36.00 & 42.4850 & 13.9677 & 42.2188 & 11.1057 & 0.3541 & 0.7520 \\
\hline 20 & 38.00 & 42.4850 & 13.9677 & 41.8710 & 10.9260 & 0.3528 & 1.7405 \\
\hline 21 & 40.00 & 42.4850 & 13.9677 & 44.0750 & 10.2090 & 0.3477 & -4.5731 \\
\hline & & & & & & & \\
\hline
\end{tabular}


Table 2 : Test of significance between $\bar{Y}$ (without missing) and $\bar{Y}$ (after imputation by ratio method)

\begin{tabular}{|c|c|c|c|c|c|c|c|}
\hline S.No. & missing \% & $\bar{Y}$ & $\begin{array}{c}\text { S.D. } \\
\text { (without) }\end{array}$ & $\begin{array}{c}\bar{Y} \\
\text { (ratio) }\end{array}$ & $\begin{array}{c}\text { S.D. } \\
\text { (ratio) }\end{array}$ & S.E. & Z-test \\
\hline 1 & 1.00 & 42.4850 & 13.9677 & 42.5149 & 13.9622 & 0.3737 & -0.0801 \\
\hline 2 & 2.00 & 42.4850 & 13.9677 & 42.3099 & 13.7705 & 0.3724 & 0.4703 \\
\hline 3 & 4.00 & 42.4850 & 13.9677 & 42.5709 & 13.8954 & 0.3732 & -0.2302 \\
\hline 4 & 6.00 & 42.4850 & 13.9677 & 42.5542 & 13.3365 & 0.3695 & -0.1873 \\
\hline 5 & 8.00 & 42.4850 & 13.9677 & 42.5850 & 13.5485 & 0.3709 & -0.2695 \\
\hline 6 & 10.00 & 42.4850 & 13.9677 & 42.0367 & 13.2146 & 0.3687 & 1.2160 \\
\hline 7 & 12.00 & 42.4850 & 13.9677 & 42.7998 & 13.0785 & 0.3677 & -0.8559 \\
\hline 8 & 14.00 & 42.4850 & 13.9677 & 42.5684 & 13.0947 & 0.3678 & -0.2268 \\
\hline 9 & 16.00 & 42.4850 & 13.9677 & 42.6550 & 12.9135 & 0.3666 & -0.4637 \\
\hline 10 & 18.00 & 42.4850 & 13.9677 & 42.3573 & 12.7720 & 0.3656 & 0.3492 \\
\hline 11 & 20.00 & 42.4850 & 13.9677 & 41.8163 & 12.2502 & 0.3621 & 1.8470 \\
\hline 12 & 22.00 & 42.4850 & 13.9677 & 42.2417 & 12.2073 & 0.3618 & 0.6726 \\
\hline 13 & 24.00 & 42.4850 & 13.9677 & 42.5645 & 12.1758 & 0.3615 & -0.2200 \\
\hline 14 & 26.00 & 42.4850 & 13.9677 & 42.5171 & 11.9701 & 0.3601 & -0.0893 \\
\hline 15 & 28.00 & 42.4850 & 13.9677 & 42.7066 & 11.6841 & 0.3581 & -0.6187 \\
\hline 16 & 30.00 & 42.4850 & 13.9677 & 42.3731 & 11.1921 & 0.3547 & 0.3154 \\
\hline 17 & 32.00 & 42.4850 & 13.9677 & 42.2630 & 12.0476 & 0.3607 & 0.6156 \\
\hline 18 & 34.00 & 42.4850 & 13.9677 & 42.4861 & 10.4015 & 0.3491 & $\mathbf{- 0 . 0 0 3 1}$ \\
\hline 19 & 36.00 & 42.4850 & 13.9677 & 42.2182 & 11.1057 & 0.3541 & 0.7534 \\
\hline 20 & 38.00 & 42.4850 & 13.9677 & 42.1737 & 10.9328 & 0.3528 & 0.8824 \\
\hline 21 & 40.00 & 42.4850 & 13.9677 & 43.5799 & 10.2270 & 0.3478 & -3.1479 \\
\hline
\end{tabular}

Table 3 : Test of significance between $\bar{Y}$ (without missing) and $\bar{Y}$ (after imputation by Compromise method)

\begin{tabular}{|c|c|c|c|c|c|c|c|}
\hline S.No. & missing \% & $\bar{Y}$ & $\begin{array}{c}\text { S.D. } \\
\text { (without) }\end{array}$ & $\begin{array}{c}\bar{Y} \\
\text { (comp) }\end{array}$ & $\begin{array}{c}\text { S.D. } \\
\text { (comp) }\end{array}$ & S.E. & Z-test \\
\hline 1 & 1.00 & 42.4850 & 13.9677 & 42.5150 & 13.9622 & 0.3737 & -0.0802 \\
\hline 2 & 2.00 & 42.4850 & 13.9677 & 42.3090 & 13.7705 & 0.3724 & 0.4727 \\
\hline 3 & 4.00 & 42.4850 & 13.9677 & 42.5726 & 13.8953 & 0.3732 & -0.2347 \\
\hline 4 & 6.00 & 42.4850 & 13.9677 & 42.5565 & 13.3365 & 0.3695 & -0.1936 \\
\hline 5 & 8.00 & 42.4850 & 13.9677 & 42.5880 & 13.5484 & 0.3709 & -0.2778 \\
\hline 6 & 10.00 & 42.4850 & 13.9677 & 42.0214 & 13.2140 & 0.3687 & 1.2575 \\
\hline 7 & 12.00 & 42.4850 & 13.9677 & 42.8051 & 13.0784 & 0.3677 & -0.8704 \\
\hline 8 & 14.00 & 42.4850 & 13.9677 & 42.5649 & 13.0947 & 0.3678 & -0.2172 \\
\hline 9 & 16.00 & 42.4850 & 13.9677 & 42.6525 & 12.9134 & 0.3666 & -0.4568 \\
\hline 10 & 18.00 & 42.4850 & 13.9677 & 42.3565 & 12.7720 & 0.3656 & 0.3514 \\
\hline 11 & 20.00 & 42.4850 & 13.9677 & 41.7926 & 12.2495 & 0.3621 & 1.9125 \\
\hline 12 & 22.00 & 42.4850 & 13.9677 & 42.2353 & 12.2073 & 0.3618 & 0.6902 \\
\hline 13 & 24.00 & 42.4850 & 13.9677 & 42.5487 & 12.1755 & 0.3615 & -0.1761 \\
\hline 14 & 26.00 & 42.4850 & 13.9677 & 42.5417 & 11.9696 & 0.3601 & -0.1574 \\
\hline 15 & 28.00 & 42.4850 & 13.9677 & 42.7033 & 11.6807 & 0.3581 & -0.6097 \\
\hline 16 & 30.00 & 42.4850 & 13.9677 & 42.3252 & 11.1903 & 0.3547 & 0.4507 \\
\hline 17 & 32.00 & 42.4850 & 13.9677 & 42.4589 & 12.1812 & 0.3616 & 0.0721 \\
\hline 18 & 34.00 & 42.4850 & 13.9677 & 42.4701 & 10.4013 & 0.3491 & $\mathbf{0 . 0 4 2 6}$ \\
\hline 19 & 36.00 & 42.4850 & 13.9677 & 42.2184 & 11.1057 & 0.3541 & 0.7531 \\
\hline 20 & 38.00 & 42.4850 & 13.9677 & 42.1205 & 10.9306 & 0.3528 & 1.0330 \\
\hline 21 & 40.00 & 42.4850 & 13.9677 & 43.7278 & 10.2178 & 0.3477 & -3.5739 \\
\hline & & & & & & & \\
\hline
\end{tabular}


Table 4 : Test of significance between $\bar{Y}_{\text {(without missing) and }} \bar{Y}_{\text {(after imputation by Ahmed method( using estimator t2) }}$

\begin{tabular}{|c|c|c|c|c|c|c|c|c|}
\hline S.No. & missing \% & $\mathbf{V}$ & $\begin{array}{c}\bar{Y} \\
\text { without } \\
\text { missing }\end{array}$ & $\begin{array}{c}\text { S.D. } \\
\text { (without) }\end{array}$ & $\begin{array}{c}\text { t2 } \\
\text { (ahmed) }\end{array}$ & $\begin{array}{c}\text { S.D. } \\
\text { (ahmed) }\end{array}$ & S.E. & Z-test \\
\hline 1 & 1.00 & 0.7643 & 42.4850 & 13.9677 & 42.5150 & 13.9622 & 0.3737 & -0.0802 \\
\hline 2 & 2.00 & 0.7722 & 42.4850 & 13.9677 & 42.3090 & 13.7705 & 0.3724 & 0.4726 \\
\hline 3 & 4.00 & 0.7683 & 42.4850 & 13.9677 & 42.5726 & 13.8953 & 0.3732 & -0.2346 \\
\hline 4 & 6.00 & 0.7573 & 42.4850 & 13.9677 & 42.5565 & 13.3365 & 0.3695 & -0.1936 \\
\hline 5 & 8.00 & 0.7608 & 42.4850 & 13.9677 & 42.5880 & 13.5484 & 0.3709 & -0.2778 \\
\hline 6 & 10.00 & 0.7820 & 42.4850 & 13.9677 & 42.0213 & 13.2140 & 0.3687 & 1.2577 \\
\hline 7 & 12.00 & 0.7785 & 42.4850 & 13.9677 & 42.8051 & 13.0784 & 0.3677 & -0.8704 \\
\hline 8 & 14.00 & 0.7818 & 42.4850 & 13.9677 & 42.5649 & 13.0947 & 0.3678 & -0.2172 \\
\hline 9 & 16.00 & 0.7925 & 42.4850 & 13.9677 & 42.6525 & 12.9134 & 0.3666 & -0.4568 \\
\hline 10 & 18.00 & 0.7791 & 42.4850 & 13.9677 & 42.3565 & 12.7720 & 0.3656 & 0.3514 \\
\hline 11 & 20.00 & 0.7716 & 42.4850 & 13.9677 & 41.7925 & 12.2495 & 0.3621 & 1.9128 \\
\hline 12 & 22.00 & 0.7885 & 42.4850 & 13.9677 & 42.2353 & 12.2073 & 0.3618 & 0.6903 \\
\hline 13 & 24.00 & 0.7957 & 42.4850 & 13.9677 & 42.5486 & 12.1755 & 0.3615 & -0.1759 \\
\hline 14 & 26.00 & 0.7794 & 42.4850 & 13.9677 & 42.5416 & 11.9696 & 0.3601 & -0.1571 \\
\hline 15 & 28.00 & 0.7706 & 42.4850 & 13.9677 & 42.6846 & 11.6836 & 0.3581 & -0.5573 \\
\hline 16 & 30.00 & 0.7704 & 42.4850 & 13.9677 & 42.3249 & 11.1903 & 0.3547 & 0.4515 \\
\hline 17 & 32.00 & 0.7820 & 42.4850 & 13.9677 & 42.4584 & 12.1812 & 0.3616 & 0.0737 \\
\hline 18 & 34.00 & 0.7929 & 42.4850 & 13.9677 & 42.4701 & 10.4013 & 0.3491 & $\mathbf{0 . 0 4 2 7}$ \\
\hline 19 & 36.00 & 0.7596 & 42.4850 & 13.9677 & 42.2184 & 11.1057 & 0.3541 & 0.7531 \\
\hline 20 & 38.00 & 0.8245 & 42.4850 & 13.9677 & 42.1201 & 10.9306 & 0.3528 & 1.0342 \\
\hline 21 & 40.00 & 0.7012 & 42.4850 & 13.9677 & 43.7264 & 10.2179 & 0.3477 & -3.5697 \\
\hline
\end{tabular}

Table 5: Test of significance between $\bar{Y}$ (without missing) and $\bar{Y}$ (after imputation by factor type estimator $T_{F T 2}$ at k1)

\begin{tabular}{|c|c|c|c|c|c|c|c|c|c|c|}
\hline S.No. & missing \% & $\mathbf{F}$ & $\mathbf{V}$ & $\mathbf{k 1}$ & $\begin{array}{c}\bar{Y} \\
\text { Without } \\
\text { missing }\end{array}$ & $\begin{array}{c}\text { S.D. } \\
\text { (without) }\end{array}$ & $T_{F T 2}$ & S.D. & S.E. & Z-test \\
\hline 1 & 1.00 & 0.99 & 0.7643 & 1.1789 & 42.4850 & 13.9677 & 42.5150 & 13.9622 & 0.3737 & -0.0802 \\
\hline 2 & 2.00 & 0.98 & 0.7722 & 1.1789 & 42.4850 & 13.9677 & 42.3090 & 13.7705 & 0.3724 & 0.4726 \\
\hline 3 & 4.00 & 0.96 & 0.7683 & 1.1799 & 42.4850 & 13.9677 & 42.5726 & 13.8953 & 0.3732 & -0.2346 \\
\hline 4 & 6.00 & 0.94 & 0.7573 & 1.1902 & 42.4850 & 13.9677 & 42.5565 & 13.3365 & 0.3695 & -0.1936 \\
\hline 5 & 8.00 & 0.92 & 0.7608 & 1.1905 & 42.4850 & 13.9677 & 42.5880 & 13.5484 & 0.3709 & -0.2778 \\
\hline 6 & 10.00 & 0.90 & 0.7820 & 1.1734 & 42.4850 & 13.9677 & 42.0218 & 13.2140 & 0.3687 & 1.2565 \\
\hline 7 & 12.00 & 0.88 & 0.7785 & 1.1835 & 42.4850 & 13.9677 & 42.8051 & 13.0784 & 0.3677 & -0.8704 \\
\hline 8 & 14.00 & 0.86 & 0.7818 & 1.1839 & 42.4850 & 13.9677 & 42.5649 & 13.0947 & 0.3678 & -0.2172 \\
\hline 9 & 16.00 & 0.84 & 0.7925 & 1.1790 & 42.4850 & 13.9677 & 42.6525 & 12.9134 & 0.3666 & -0.4568 \\
\hline 10 & 18.00 & 0.82 & 0.7791 & 1.1918 & 42.4850 & 13.9677 & 42.3565 & 12.7720 & 0.3656 & 0.3514 \\
\hline 11 & 20.00 & 0.80 & 0.7716 & 1.2006 & 42.4850 & 13.9677 & 41.7924 & 12.2495 & 0.3621 & 1.9128 \\
\hline 12 & 22.00 & 0.78 & 0.7885 & 1.1908 & 42.4850 & 13.9677 & 42.2353 & 12.2073 & 0.3618 & 0.6903 \\
\hline 13 & 24.00 & 0.76 & 0.7957 & 1.1884 & 42.4850 & 13.9677 & 42.5486 & 12.1755 & 0.3615 & -0.1759 \\
\hline 14 & 26.00 & 0.74 & 0.7794 & 1.2047 & 42.4850 & 13.9677 & 42.5416 & 11.9696 & 0.3601 & -0.1571 \\
\hline 15 & 28.00 & 0.72 & 0.7706 & 1.2153 & 42.4850 & 13.9677 & 42.6846 & 11.6836 & 0.3581 & -0.5573 \\
\hline 16 & 30.00 & 0.70 & 0.7704 & 1.2193 & 42.4850 & 13.9677 & 42.3248 & 11.1903 & 0.3547 & 0.4517 \\
\hline 17 & 32.00 & 0.68 & 0.7820 & 1.2137 & 42.4850 & 13.9677 & 42.4583 & 12.1812 & 0.3616 & 0.0739 \\
\hline 18 & 34.00 & 0.66 & 0.7929 & 1.2085 & 42.4850 & 13.9677 & 42.4701 & 10.4013 & 0.3491 & $\mathbf{0 . 0 4 2 7}$ \\
\hline 19 & 36.00 & 0.64 & 0.7596 & 1.2406 & 42.4850 & 13.9677 & 42.2184 & 11.1057 & 0.3541 & 0.7531 \\
\hline 20 & 38.00 & 0.62 & 0.8245 & 1.1885 & 42.4850 & 13.9677 & 42.1200 & 10.9306 & 0.3528 & 1.0344 \\
\hline 21 & 40.00 & 0.60 & 0.7012 & 1.2972 & 42.4850 & 13.9677 & 43.7295 & 10.2178 & 0.3477 & -3.5788 \\
\hline
\end{tabular}


Table 6: Test of significance between $\bar{Y}$ (without missing) and $\bar{Y}$ (after imputation by factor type estimator $T_{F T 2 \text { at }}$ k2)

\begin{tabular}{|c|c|c|c|c|c|c|c|c|c|c|}
\hline S.No. & missing \% & $\mathbf{F}$ & $\mathbf{V}$ & k2 & $\begin{array}{c}\bar{Y} \\
\text { without } \\
\text { missing }\end{array}$ & $\begin{array}{c}\text { S.D. } \\
\text { (without) }\end{array}$ & $T_{F T 2}$ & S.D. & S.E. & Z-test \\
\hline 1 & 1.00 & 0.99 & 0.7643 & 3.3782 & 42.4850 & 13.9677 & 42.5157 & 13.9622 & 0.3737 & -0.0821 \\
\hline 2 & 2.00 & 0.98 & 0.7722 & 3.3782 & 42.4850 & 13.9677 & 42.3089 & 13.7705 & 0.3724 & 0.4729 \\
\hline 3 & 4.00 & 0.96 & 0.7683 & 3.3644 & 42.4850 & 13.9677 & 42.5726 & 13.8953 & 0.3732 & -0.2346 \\
\hline 4 & 6.00 & 0.94 & 0.7573 & 3.3578 & 42.4850 & 13.9677 & 42.5565 & 13.3365 & 0.3695 & -0.1935 \\
\hline 5 & 8.00 & 0.92 & 0.7608 & 3.3474 & 42.4850 & 13.9677 & 42.5880 & 13.5484 & 0.3709 & -0.2776 \\
\hline 6 & 10.00 & 0.90 & 0.7820 & 3.4727 & 42.4850 & 13.9677 & 41.9930 & 13.2132 & 0.3687 & 1.3345 \\
\hline 7 & 12.00 & 0.88 & 0.7785 & 3.3239 & 42.4850 & 13.9677 & 42.8050 & 13.0784 & 0.3677 & -0.8702 \\
\hline 8 & 14.00 & 0.86 & 0.7818 & 3.3123 & 42.4850 & 13.9677 & 42.5649 & 13.0947 & 0.3678 & -0.2172 \\
\hline 9 & 16.00 & 0.84 & 0.7925 & 3.2987 & 42.4850 & 13.9677 & 42.6525 & 12.9134 & 0.3666 & -0.4569 \\
\hline 10 & 18.00 & 0.82 & 0.7791 & 3.2921 & 42.4850 & 13.9677 & 42.3565 & 12.7720 & 0.3656 & 0.3514 \\
\hline 11 & 20.00 & 0.80 & 0.7716 & 3.2827 & 42.4850 & 13.9677 & 41.7916 & 12.2495 & 0.3621 & 1.9153 \\
\hline 12 & 22.00 & 0.78 & 0.7885 & 3.2668 & 42.4850 & 13.9677 & 42.2352 & 12.2073 & 0.3618 & 0.6904 \\
\hline 13 & 24.00 & 0.76 & 0.7957 & 3.2536 & 42.4850 & 13.9677 & 42.5481 & 12.1755 & 0.3615 & -0.1744 \\
\hline 14 & 26.00 & 0.74 & 0.7794 & 3.2456 & 42.4850 & 13.9677 & 42.5407 & 11.9696 & 0.3601 & -0.1546 \\
\hline 15 & 28.00 & 0.72 & 0.7706 & 3.2356 & 42.4850 & 13.9677 & 42.6839 & 11.6836 & 0.3581 & -0.5554 \\
\hline 16 & 30.00 & 0.70 & 0.7704 & 3.2224 & 42.4850 & 13.9677 & 42.3221 & 11.1902 & 0.3547 & 0.4592 \\
\hline 17 & 32.00 & 0.68 & 0.7820 & 3.2058 & 42.4850 & 13.9677 & 42.4530 & 12.1814 & 0.3616 & 0.0886 \\
\hline 18 & 34.00 & 0.66 & 0.7929 & 3.1888 & 42.4850 & 13.9677 & 42.4698 & 10.4013 & 0.3491 & 0.0436 \\
\hline 19 & 36.00 & 0.64 & 0.7596 & 3.1840 & 42.4850 & 13.9677 & 42.2184 & 11.1057 & 0.3541 & 0.7531 \\
\hline 20 & 38.00 & 0.62 & 0.8245 & 3.1507 & 42.4850 & 13.9677 & 42.1153 & 10.9304 & 0.3528 & 1.0478 \\
\hline 21 & 40.00 & 0.60 & 0.7012 & 3.1722 & 42.4850 & 13.9677 & 43.7165 & 10.2184 & 0.3478 & -3.5414 \\
\hline
\end{tabular}

Table 7: Test of significance between $\bar{Y}$ (without missing) and $\bar{Y}$ (after imputation by factor type estimator $T_{F T 2}$ at k3)

\begin{tabular}{|c|c|c|c|c|c|c|c|c|c|c|}
\hline S.No. & $\begin{array}{c}\text { missing } \\
\text { \% }\end{array}$ & $\mathbf{f}$ & $\mathbf{V}$ & $\mathbf{k 3}$ & $\begin{array}{c}\bar{Y} \\
\text { without } \\
\text { missing }\end{array}$ & $\begin{array}{c}\text { S.D. } \\
\text { (without) }\end{array}$ & $T_{F T 2}$ & S.D. & S.E. & Z-test \\
\hline 1 & 1.00 & 0.99 & 0.7643 & 15.0965 & 42.4850 & 13.9677 & 42.5150 & 13.9622 & 0.3737 & -0.0803 \\
\hline 2 & 2.00 & 0.98 & 0.7722 & 15.0965 & 42.4850 & 13.9677 & 42.3090 & 13.7705 & 0.3724 & 0.4727 \\
\hline 3 & 4.00 & 0.96 & 0.7683 & 15.0978 & 42.4850 & 13.9677 & 42.5726 & 13.8953 & 0.3732 & -0.2346 \\
\hline 4 & 6.00 & 0.94 & 0.7573 & 14.3794 & 42.4850 & 13.9677 & 42.5565 & 13.3365 & 0.3695 & -0.1936 \\
\hline 5 & 8.00 & 0.92 & 0.7608 & 14.4220 & 42.4850 & 13.9677 & 42.5880 & 13.5484 & 0.3709 & -0.2778 \\
\hline 6 & 10.00 & 0.90 & 0.7820 & 13.6228 & 42.4850 & 13.9677 & 42.0188 & 13.2139 & 0.3687 & 1.2645 \\
\hline 7 & 12.00 & 0.88 & 0.7785 & 15.0732 & 42.4850 & 13.9677 & 42.8051 & 13.0784 & 0.3677 & -0.8704 \\
\hline 8 & 14.00 & 0.86 & 0.7818 & 15.1178 & 42.4850 & 13.9677 & 42.5649 & 13.0947 & 0.3678 & -0.2172 \\
\hline 9 & 16.00 & 0.84 & 0.7925 & 15.5984 & 42.4850 & 13.9677 & 42.6525 & 12.9134 & 0.3666 & -0.4568 \\
\hline 10 & 18.00 & 0.82 & 0.7791 & 14.6478 & 42.4850 & 13.9677 & 42.3565 & 12.7720 & 0.3656 & 0.3514 \\
\hline 11 & 20.00 & 0.80 & 0.7716 & 14.1015 & 42.4850 & 13.9677 & 41.7924 & 12.2495 & 0.3621 & 1.9129 \\
\hline 12 & 22.00 & 0.78 & 0.7885 & 14.8744 & 42.4850 & 13.9677 & 42.2353 & 12.2073 & 0.3618 & 0.6903 \\
\hline 13 & 24.00 & 0.76 & 0.7957 & 15.1336 & 42.4850 & 13.9677 & 42.5486 & 12.1755 & 0.3615 & -0.1759 \\
\hline 14 & 26.00 & 0.74 & 0.7794 & 14.0511 & 42.4850 & 13.9677 & 42.5415 & 11.9696 & 0.3601 & -0.1570 \\
\hline 15 & 28.00 & 0.72 & 0.7706 & 13.4643 & 42.4850 & 13.9677 & 42.6846 & 11.6836 & 0.3581 & -0.5572 \\
\hline 16 & 30.00 & 0.70 & 0.7704 & 13.3099 & 42.4850 & 13.9677 & 42.3247 & 11.1903 & 0.3547 & 0.4519 \\
\hline 17 & 32.00 & 0.68 & 0.7820 & 13.7266 & 42.4850 & 13.9677 & 42.4581 & 12.1812 & 0.3616 & 0.0744 \\
\hline
\end{tabular}




\begin{tabular}{|l|l|l|l|l|l|l|l|l|l|l|}
\hline 18 & 34.00 & 0.66 & 0.7929 & 14.1445 & 42.4850 & 13.9677 & 42.4701 & 10.4013 & 0.3491 & $\mathbf{0 . 0 4 2 7}$ \\
\hline 19 & 36.00 & 0.64 & 0.7596 & 12.4188 & 42.4850 & 13.9677 & 42.2184 & 11.1057 & 0.3541 & 0.7531 \\
\hline 20 & 38.00 & 0.62 & 0.8245 & 15.8030 & 42.4850 & 13.9677 & 42.1199 & 10.9306 & 0.3528 & 1.0347 \\
\hline 21 & 40.00 & 0.60 & 0.7012 & 10.2930 & 42.4850 & 13.9677 & 43.7256 & 10.2180 & 0.3477 & -3.5675 \\
\hline
\end{tabular}

\section{CONCLUSION}

This work analyses the behavior of five imputation methods that can be used for missing data treatment. These methods are analyzed on different percentages of missing data into a common attribute of large data sets. The Ratio method of imputation and Factor type compromised method of imputation provides very good results, even for training sets having a large amount of missing data. In case of mean method of imputation, only at $24 \%$ level of missing data, critical value of $\mathrm{z}$ score i.e. 0.0051 is less than $5 \%$ level of significance which shows that the results are almost same in case of mean of attribute domain (without missing) and mean attribute domain(with missing) at this percent. In case of ratio method of imputation and, only at $34 \%$ level of missing data, critical value of $\mathrm{z}$ score i.e. 0.0031 is less than $5 \%$ level of significance which shows that the results are almost same in case of mean of attribute domain (without missing) and mean attribute domain(with missing) at this percent. In case of Ahmed method of imputation, only at $34 \%$ level of missing data, critical value of $\mathrm{z}$ score i.e. 0.0427 is less than $5 \%$ level of significance which shows that the results are almost same in case of mean of attribute domain (without missing) and mean attribute domain(with missing) at this percent. In case of Factor type compromised method of imputation, at 34\% level of missing data, critical value of $\mathrm{z}$ score's are 0.0427, $0.04276 \& 0.0427$ respectively that is less than $5 \%$ level of significance which shows that the results are almost same in case of mean of attribute domain (without missing) and mean attribute domain(with missing) at this percent.

Although, all the methods are showing approximately correct results at different percentages of missing data but when we compare results of all the methods on same data set, outcome given by ratio method of imputation and Factor type compromised method are more accurate among all. Hence, it may be recommended for imputing the missing values to preprocess the database prior to analysis, so that the quality of the results extracted can be improved.

In future works, the missing data treatment methods will be analyzed in other data sets. Furthermore, in this work missing values were inserted completely at random (MCAR). In a future work, one canl analyze the behavior of these methods when missing values are not randomly distributed. In this case, there is a possibility of creating invalid knowledge. For an effective analysis, it is recommended to inspect not only the error rate, but also the quality of the knowledge induced by the learning system.

\section{REFERENCES}

[1] Ahmed, M. S., Al-Titi, O., Al-Rawi, Z. and Abu-Dayyeh, W. 2006. Estimation of a population mean using different imputation methods, Statistics in Transition, 7, $6,1247-1264$.

[2] Cochran, W. G. 2005. Sampling Techniques, John Wiley and Sons, New York.
[3] G. E. A. P. A. Batista and M. C. Monard. K-Nearest Neighbour as Imputation Method 2002. Experimental Results. Technical report, ICMC-USP, ISSN-0103-2569.

[4] Heitjan, D. F. and Basu, S. 1996. Distinguishing 'Missing at random' and 'missing completely at random', The American Statistician, 50, 207-213.

[5] J. W. Grzymala-Busse and M. Hu. A Comparison of Several Approaches to Missing Attribute Values in Data Mining 2000. In RSCTC'2000, pages 340-347.

[6] K. Lakshminarayan, S. A. Harp, and T. Samad. 1999. Imputation of Missing Data in Industrial Databases. Applied Intelligence, 11:259-275.

[7] R. J. Little and D. B. Rubin. 1987. Statistical Analysis with Missing Data. John Wiley and Sons, New York, 1987.

[8] Rao, J. N. K. and Sitter, R. R. 1995. Variance estimation under two-phase sampling with application to imputation for missing data, Biometrica, 82, 453-460.

[9] Reddy, V. N. 1978. A study on the use of prior knowledge on certain population parameters in estimation, Sankhya, C, 40, 29-37.

[10] Rubin, D. B. 1976. Inference and missing data, Biometrica, 63, 581-593.

[11] Shukla, D. 2002. F-T estimator under two-phase sampling, Metron, 59, 1-2, 253-263.

[12] Shukla, D. and Thakur, N. S. 2008. Estimation of mean with imputation of missing data using factor-type estimator, Statistics in Transition, 9, 1, 33-48.

[13] Thakur, N. S., Yadav Kalpana, and Pathak S. 2012. Some imputation methods in double sampling scheme for estimation of population mean, IJMER, Vol.2, Issue.1 Jan-Feb 2012 pp-200-207.

[14] Thakur, N. S., Yadav Kalpana, and Pathak S. 2011.Estimation of mean in presence of missingdata under two-phase sampling scheme, JRSS,Vol 4, issue 2,93-104.

[15] Singh, S. 2009. A new method of imputation in survey sampling, Statistics, Vol. 43, 5 , 499 - 511.

[16] Singh, S. and Horn, S. 2000. Compromised imputation in survey sampling, Metrika, 51, 266-276.

[17] Singh, V. K. and Shukla, D. 1993. An efficient one parameter family of factor - type estimator in sample survey, Metron, 51, 1-2, 139-159.

[18] Singhai, R 2013. Comparative Study of Three Imputation Methods to Treat Missing Values, IJCT, Council of Inovative Research, 2013. 